\title{
Crystal chemistry of the mendipite-type system $\mathrm{Pb}_{3} \mathrm{O}_{2} \mathrm{Cl}_{2}-\mathrm{Pb}_{3} \mathrm{O}_{2} \mathrm{Br}_{2}$
}

\author{
Oleg I. Siidra*, I, Sergey V. Krivovichev ${ }^{\mathrm{I}}$, Thomas Armbruster ${ }^{\mathrm{II}}$ and Wulf Depmeier ${ }^{\mathrm{III}}$ \\ I Department of Crystallography, St. Petersburg State University, University Emb. 7/9, 199034 St. Petersburg, Russia \\ II Laboratorium für chemische und mineralogische Kristallographie, Universität Bern, Freiestraße 3, 3102 Bern, Switzerland \\ III Institut für Geowissenschaften, Universität zu Kiel, Olshausenstraße 40, 24118 Kiel, Germany
}

Received September 10, 2007; accepted December 18, 2007

\section{Lead oxyhalides / Mendipite / Oxocentered tetrahedra / Conformation / Single crystal structure analysis / $X$-ray diffraction}

\begin{abstract}
The crystal structures of the mendipite series $\mathrm{Pb}_{3} \mathrm{O}_{2} \mathrm{Cl}_{2}-\mathrm{Pb}_{3} \mathrm{O}_{2} \mathrm{Br}_{2}$ have been refined. The structures are based upon $\left[\mathrm{O}_{2} \mathrm{~Pb}_{3}\right]^{2+}$ double chains of edge-sharing $\mathrm{OPb}_{4}$ tetrahedra. There are three symmetrically independent $\mathrm{Pb}^{2+}$ cations. The number of nonequivalent halogen sites is two (X1, X2). Short $\mathrm{Pb}-\mathrm{O}$ bonds are located on one side of the $\mathrm{Pb}^{2+}$ cations and weak $\mathrm{Pb}-\mathrm{X}$ bonds are located on the other side of the $\mathrm{Pb}^{2+}$ coordination sphere. The evident strong distortion of the $\mathrm{Pb}^{2+}$ coordination polyhedra is due to the stereoactivity of the $6 s^{2}$ lone electron pairs of the $\mathrm{Pb}^{2+}$ cations. $\mathrm{Pb} 1-\mathrm{X} 2$ and $\mathrm{Pb} 2-\mathrm{X} 2$ bonds are the most sensitive to the $\mathrm{X}$ site occupancy, which is in agreement with the non-linear behavior of the $a$ and $c$ parameters. Determination of unit-cell parameters by single crystal studies showed strong deviation from Vegard's rule. Nonlinearity of the lattice parameters is caused by selective ordering of the halide anions over $\mathrm{X} 1$ and $\mathrm{X} 2$ sites. $\mathrm{Br}$ atoms prefer the $\mathrm{X} 2$ position, whereas $\mathrm{Cl}$ prefers the $\mathrm{X} 1$ site. The angle between two adjacent $\mathrm{OPb}_{4}$ tetrahedra was determined to analyze the influence of halogen atoms on the structure of the $\left[\mathrm{O}_{2} \mathrm{~Pb}_{3}\right]^{2+}$ chain. Different occupancy of the $\mathrm{X} 1$ site by $\mathrm{Cl}$ and $\mathrm{Br}$ atoms leads to most pronounced angular changes. These observations may be interpreted as adaptation of the $\left[\mathrm{O}_{2} \mathrm{~Pb}_{3}\right]^{2+}$ double chains to the large halide ions in the crystal structures of the mendipite series compounds.
\end{abstract}

\section{Introduction}

Mendipite $\mathrm{Pb}_{3} \mathrm{O}_{2} \mathrm{Cl}_{2}$ is the most abundant mineral among the natural lead oxyhalides. It was first described by Spencer and Mountain (1923) from the Mendip Hills, England. The crystal structure was solved by Gabrielson (1957) and refined in $P 2{ }_{1} 2_{1} 2_{1}$ space group. Later, Vincent and Perrault (1971) refined the crystal structure of synthetic $\mathrm{Pb}_{3} \mathrm{O}_{2} \mathrm{Cl}_{2}$ in Pnma. In the same space group, the structure of natural mendipite was refined by Pasero and Vacchiano

* Correspondence author (e-mail: siidra@mail.ru)
(2000). The most recent single-crystal studies of synthetic $\mathrm{Pb}_{3} \mathrm{O}_{2} \mathrm{Cl}_{2}$ were published by Krivovichev and Burns (2001). The crystal structure of $\mathrm{Pb}_{3} \mathrm{O}_{2} \mathrm{Br}_{2}$ was determined using powder samples by Berdonosov et al. (1996) and later by Noren et al. (2002).

The environmental importance of lead oxyhalides was pointed out by many authors. $\mathrm{Pb}$ oxychlorides were detected in dust particles emitted from a lead smelter (Sobanska et al., 1999; Wu, Biswas, 2000). Pb halides (chloride-bromides) as well as oxy- and hydroxyhalides were observed in automobile exhaust gases (Post, Buseck, 1985) and roadside soils (Smith, 1976). However, $\mathrm{Pb}$ oxyhalides have not only environmental importance and are also of interest from the viewpoint of material science as anisotropic materials with a variety of physical properties that depend strongly on the crystallographic direction. Sigman and Korgel (2005) recently described the synthesis and properties of highly birefringent nanostructures with mendipite composition. To date, detailed chemical and structural information is available for pure oxy- and hydroxychloride (Krivovichev, Burns, 2001a; 2002; 2006; Siidra et al., 2007 a, b, c), oxy- and hydroxybromide (Krivovichev, Burns, 2001b; Siidra et al., 2007d; Keller, 1983; Riebe, Keller, 1989) and oxyiodide (Welch et al., 2001) systems, whereas little is known about mixed halide systems such as $\mathrm{Cl}-\mathrm{Br}$ (Krivovichev et al., 2006).

The practical importance of mendipite-related phases prompted us to take a closer look at the crystal chemistry of the $\mathrm{Pb}_{3} \mathrm{O}_{2} \mathrm{Cl}_{2}-\mathrm{Pb}_{3} \mathrm{O}_{2} \mathrm{Br}_{2}$ system. Single-crystal studies of these phases also provide important information pertinent to an understanding of the transport of lead and the crystallization of lead compounds in natural systems.

\section{Experimental}

\section{Synthesis}

Single crystals of mendipite phases were obtained by the solid-state reactions method. $\mathrm{PbO}$ (Merck, 99\%), $\mathrm{PbCl}_{2}$ (Aldrich, 99,9\%) and $\mathrm{PbBr}_{2}$ (Aldrich, 99,9\%) were used as received. Reactants were mixed in an agate mortar in quantities according to the given stoichiometry. Syntheses were carried out with increment of $10 \%$ in the $\mathrm{Cl}: \mathrm{Br}$ ratio 
Table 1. Crystallographic data and chemical composition of mendipite-related compounds.

\begin{tabular}{|c|c|c|c|c|c|c|c|}
\hline Sample $^{a}$ & $\mathrm{Cl}: \mathrm{Br}^{b}$ & Formula $^{c}$ & Formula $^{d}$ & $a, \AA$ & $b, \AA$ & $c, \AA$ & $V, \AA^{3}$ \\
\hline M1 & $0: 1$ & $\mathrm{~Pb}_{3.04} \mathrm{O}_{2} \mathrm{Br}_{1.92}$ & $\mathrm{~Pb}_{3} \mathrm{O}_{2} \mathrm{Br}_{2}$ & $12.244(5)$ & $5.872(2)$ & $9.799(4)$ & $704.6(5)$ \\
\hline M2 & $1: 9$ & $\mathrm{~Pb}_{2.98} \mathrm{O}_{2}\left(\mathrm{Cl}_{0.15} \mathrm{Br}_{1.86}\right)_{2.01}$ & $\mathrm{~Pb}_{3} \mathrm{O}_{2} \mathrm{Cl}_{0.19} \mathrm{Br}_{1.81}$ & $12.1949(7)$ & $5.8705(5)$ & $9.7968(9)$ & 701.4(7) \\
\hline M3 & $2: 8$ & $\mathrm{~Pb}_{2.98} \mathrm{O}_{2}\left(\mathrm{Cl}_{0.38} \mathrm{Br}_{1.63}\right)_{2.01}$ & $\mathrm{~Pb}_{3} \mathrm{O}_{2} \mathrm{Cl}_{0.46} \mathrm{Br}_{1.54}$ & $12.100(9)$ & $5.855(5)$ & $9.755(2)$ & $691.1(8)$ \\
\hline M4 & $3: 7$ & $\mathrm{~Pb}_{3.02} \mathrm{O}_{2}\left(\mathrm{Cl}_{0.56} \mathrm{Br}_{1.42}\right)_{1.98}$ & $\mathrm{~Pb}_{3} \mathrm{O}_{2} \mathrm{Cl}_{0.52} \mathrm{Br}_{1.48}$ & $12.0518(11)$ & $5.8556(5)$ & $9.7526(9)$ & $688.25(11)$ \\
\hline M5 & $4: 6$ & $\mathrm{~Pb}_{3.03} \mathrm{O}_{2}\left(\mathrm{Cl}_{0.79} \mathrm{Br}_{1.18}\right)_{1.97}$ & $\mathrm{~Pb}_{3} \mathrm{O}_{2} \mathrm{Cl}_{0.81} \mathrm{Br}_{1.19}$ & 11.9818(19) & $5.8485(9)$ & $9.7273(15)$ & $681.65(18)$ \\
\hline M6 & $5: 5$ & $\mathrm{~Pb}_{3.02} \mathrm{O}_{2}\left(\mathrm{Cl}_{0.94} \mathrm{Br}_{1.04}\right)_{1.98}$ & $\mathrm{~Pb}_{3} \mathrm{O}_{2} \mathrm{Cl}_{1.03} \mathrm{Br}_{0.97}$ & $11.922(5)$ & $5.835(2)$ & $9.701(4)$ & $674.8(4)$ \\
\hline M7 & $6: 4$ & $\mathrm{~Pb}_{3.01} \mathrm{O}_{2}\left(\mathrm{Cl}_{1.17} \mathrm{Br}_{0.82}\right)_{1.99}$ & $\mathrm{~Pb}_{3} \mathrm{O}_{2} \mathrm{Cl}_{1.09} \mathrm{Br}_{0.91}$ & $11.917(9)$ & $5.819(5)$ & $9.663(8)$ & $670.1(9)$ \\
\hline M8 & $7: 3$ & $\mathrm{~Pb}_{2.98} \mathrm{O}_{2}\left(\mathrm{Cl}_{1.39} \mathrm{Br}_{0.63}\right)_{2.02}$ & $\mathrm{~Pb}_{3} \mathrm{O}_{2} \mathrm{Cl}_{1.41} \mathrm{Br}_{0.59}$ & $11.8957(28)$ & $5.8244(14)$ & $9.6441(23)$ & $668.2(3)$ \\
\hline M9 & $8: 2$ & $\mathrm{~Pb}_{3.01} \mathrm{O}_{2}\left(\mathrm{Cl}_{1.61} \mathrm{Br}_{0.41}\right)_{2.02}$ & $\mathrm{~Pb}_{3} \mathrm{O}_{2} \mathrm{Cl}_{1.61} \mathrm{Br}_{0.39}$ & $11.9077(17)$ & $5.8264(8)$ & $9.6117(13)$ & $666.85(16)$ \\
\hline M10 & $9: 1$ & $\mathrm{~Pb}_{3.02} \mathrm{O}_{2}\left(\mathrm{Cl}_{1.76} \mathrm{Br}_{0.22}\right)_{1.98}$ & $\mathrm{~Pb}_{3} \mathrm{O}_{2} \mathrm{Cl}_{1.84} \mathrm{Br}_{0.16}$ & $11.8928(17)$ & $5.8163(9)$ & $9.5653(14)$ & $661.65(17)$ \\
\hline M11 & $1: 0$ & $\mathrm{~Pb}_{3.02} \mathrm{O}_{2} \mathrm{Cl}_{1.98}$ & $\mathrm{~Pb}_{3} \mathrm{O}_{2} \mathrm{Cl}_{2}$ & $11.808(8)$ & $5.7790(41)$ & $9.4784(68)$ & $646.8(8)$ \\
\hline
\end{tabular}

a: $\mathrm{M}=$ mendipite; $\mathrm{b}: \mathrm{Cl}: \mathrm{Br}$ ratio used in the synthesis; c: obtained by electron microprobe analysis; d: obtained by single-crystal $\mathrm{X}$-ray analysis

(Table 1). Syntheses marked in this table as M1 and M11 are bromine- and chlorine end-member of the $\mathrm{Pb}_{3} \mathrm{O}_{2} \mathrm{Cl}_{2}-$ $\mathrm{Pb}_{3} \mathrm{O}_{2} \mathrm{Br}_{2}$ series, respectively. The produced stoichiometric mixtures were loaded into platinum crucibles and heated in a furnace Carbolite 1200. The following syntheses conditions were found to be optimal to grow single crystals suitable for single crystal X-ray diffraction studies: 1) keeping the mixture at $715^{\circ} \mathrm{C}$ for $1 \mathrm{~h}$ in air; 2) cooling to $685^{\circ} \mathrm{C}$ with a cooling rate of $1^{\circ} \mathrm{C} \mathrm{min}^{-1}$ and keeping at this temperature for $15 \mathrm{~min}$; 3 ) cooling down to room temperature with a cooling rate of $30{ }^{\circ} \mathrm{C} \mathrm{h}^{-1}$. The products of the syntheses consisted of yellowish elongated transparent crystals up to $10 \mathrm{~mm}$ in size. Powder diffraction patterns were obtained with a Siemens XP18 2 diffractometer for each synthesis to confirm the absence of any by-products. Quantitative electron microprobe analysis provided the chemical formulas for all synthesis products (Table 1). Camscan-4DV electron-scan microscope and AN-10000 semiconductor spectrometer were used at $20 \mathrm{kV}$ and $0.7 \mathrm{nA}$. PbL $\alpha, \operatorname{BrL} \alpha, \mathrm{ClK} \alpha$ were used as analytic lines. Spectrum of the $\mathrm{PbM} \alpha$ line was subtracted to get the correct chemical formula because of its overlapping with the $\mathrm{ClKa}$ line. $\mathrm{PbCl}_{2}$ and $\mathrm{PbBr}_{2}$ were used as standards. All calculations were made using AF4/FLS software.

\section{X-ray data collection}

Suitable crystals of the mendipite-related compounds were mounted on a Bruker $1 \mathrm{~K}$ three-circle CCD based X-ray diffractometer operated at $50 \mathrm{kV}$ and $40 \mathrm{~mA}$. More than a hemisphere of three-dimensional data was collected for each crystal using monochromatic $\mathrm{Mo}_{\alpha} \mathrm{X}$-radiation, with frame widths of $0.3^{\circ}$ in $2 \theta$, and with 20 seconds spent counting for each frame. The unit-cell parameters were refined using least-squares techniques. The intensity data were integrated and corrected for Lorentz, polarization, and background effects using the Bruker program SAINT. All the crystals were modeled as ellipsoids for semi-empirical absorption-corrections.

\section{Structure solution and refinement}

SHELX-97 programs within the WINGX package were used for the determination and refinement of the structures of all compounds (Table 2). The refinements were made on the basis of parameters taken from Krivovichev and Burns (2001a). The final models included atomic positional parameters and anisotropic-displacement parameters for all atoms, and a weighting scheme of the structure

Table 2. Crystallographic data and refinement parameters for mendipite-related compounds.

\begin{tabular}{|c|c|c|c|c|c|c|c|c|c|}
\hline Sample & $\begin{array}{l}\text { Crystal size } \\
(\mathrm{mm})\end{array}$ & $F(000)$ & $\begin{array}{l}\text { Total } \\
\text { reflections }\end{array}$ & $\begin{array}{l}\text { Unique } \\
\left|F_{0}\right| \geq 4 \mathrm{~s}_{\mathrm{F}}\end{array}$ & $2 T_{\max }$ & GooF & $\begin{array}{l}D_{\text {calc }} \\
\left(\mathrm{g} / \mathrm{cm}^{3}\right)\end{array}$ & $R_{1}$ & $w R_{2}$ \\
\hline M1 & $0.10 \times 0.03 \times 0.05$ & 1328 & 3848 & 596 & 27.98 & 0.931 & 7.668 & 0.056 & 0.093 \\
\hline M2 & $0.08 \times 0.01 \times 0.01$ & 1315 & 3735 & 770 & 27.81 & 1.069 & 7.624 & 0.065 & 0.069 \\
\hline M3 & $0.07 \times 0.01 \times 0.02$ & 1295 & 3061 & 565 & 26.98 & 1.091 & 7.622 & 0.081 & 0.121 \\
\hline M4 & $0.09 \times 0.01 \times 0.01$ & 1284 & 3720 & 754 & 28.07 & 1.039 & 7.585 & 0.025 & 0.031 \\
\hline M5 & $0.08 \times 0.04 \times 0.02$ & 1270 & 3561 & 746 & 27.68 & 1.100 & 7.576 & 0.047 & 0.051 \\
\hline M6 & $0.07 \times 0.02 \times 0.01$ & 1253 & 3638 & 622 & 27.95 & 0.972 & 7.553 & 0.083 & 0.104 \\
\hline M7 & $0.09 \times 0.01 \times 0.01$ & 1250 & 3428 & 639 & 27.78 & 1.028 & 7.584 & 0.053 & 0.072 \\
\hline M8 & $0.08 \times 0.03 \times 0.01$ & 1227 & 3566 & 722 & 27.89 & 1.068 & 7.467 & 0.038 & 0.044 \\
\hline M9 & $0.08 \times 0.02 \times 0.01$ & 1212 & 3608 & 739 & 28.06 & 1.105 & 7.388 & 0.051 & 0.055 \\
\hline M10 & $0.09 \times 0.05 \times 0.04$ & 1198 & 3532 & 678 & 27.77 & 1.054 & 7.361 & 0.053 & 0.061 \\
\hline M11 & $0.06 \times 0.01 \times 0.01$ & 1184 & 3551 & 583 & 28.00 & 0.926 & 7.440 & 0.040 & 0.069 \\
\hline
\end{tabular}




\begin{tabular}{|c|c|c|c|c|c|c|}
\hline Atom & Sample & $x$ & $y$ & $z$ & $U_{\mathrm{eq}}, \AA^{2}$ & Occupancy \\
\hline \multirow[t]{11}{*}{$\mathrm{Pb} 1$} & M1 & $0.42101(11)$ & 0.2500 & $0.42105(11)$ & $0.0161(4)$ & \\
\hline & M2 & $0.42100(7)$ & 0.2500 & $0.42054(10)$ & $0.0157(4)$ & \\
\hline & M3 & $0.42058(15)$ & 0.2500 & $0.41974(16)$ & $0.0156(6)$ & \\
\hline & M4 & $0.42055(3)$ & 0.2500 & $0.41893(4)$ & $0.01764(15)$ & \\
\hline & M5 & $0.42032(5)$ & 0.2500 & $0.41794(7)$ & $0.0172(3)$ & \\
\hline & M6 & $0.42017(14)$ & 0.2500 & $0.41750(16)$ & $0.0150(6)$ & \\
\hline & M7 & $0.41996(9)$ & 0.2500 & $0.41742(10)$ & $0.0166(4)$ & \\
\hline & M8 & $0.41965(5)$ & 0.2500 & $0.41734(6)$ & $0.0192(2)$ & \\
\hline & M9 & $0.41928(5)$ & 0.2500 & $0.41756(8)$ & $0.0162(3)$ & \\
\hline & M10 & $0.41890(8)$ & 0.2500 & $0.41778(9)$ & $0.0157(4)$ & \\
\hline & M11 & $0.41861(7)$ & 0.2500 & $0.41795(10)$ & $0.0149(3)$ & \\
\hline \multirow[t]{11}{*}{$\mathrm{Pb} 2$} & M1 & $0.71266(12)$ & 0.2500 & $0.36806(12)$ & $0.0196(4)$ & \\
\hline & M2 & $0.71352(8)$ & 0.2500 & $0.36924(12)$ & $0.0190(4)$ & \\
\hline & M3 & $0.71497(15)$ & 0.2500 & $0.37037(19)$ & $0.0189(7)$ & \\
\hline & M4 & $0.71677(4)$ & 0.2500 & $0.37223(5)$ & $0.02139(16)$ & \\
\hline & M5 & $0.71849(5)$ & 0.2500 & $0.37371(8)$ & $0.0209(3)$ & \\
\hline & M6 & $0.71929(14)$ & 0.2500 & $0.37437(19)$ & $0.0189(6)$ & \\
\hline & M7 & $0.71973(10)$ & 0.2500 & $0.37392(11)$ & $0.0206(4)$ & \\
\hline & M8 & $0.71953(5)$ & 0.2500 & $0.37297(7)$ & $0.0231(2)$ & \\
\hline & M9 & $0.71917(6)$ & 0.2500 & $0.37150(9)$ & $0.0202(3)$ & \\
\hline & M10 & $0.71874(8)$ & 0.2500 & $0.37002(10)$ & $0.0194(4)$ & \\
\hline & M11 & $0.71822(7)$ & 0.2500 & $0.36829(10)$ & $0.0185(3)$ & \\
\hline \multirow[t]{11}{*}{$\mathrm{Pb} 3$} & M1 & $0.44199(12)$ & 0.2500 & $0.78803(11)$ & $0.0188(4)$ & \\
\hline & M2 & $0.44186(8)$ & 0.2500 & $0.78784(10)$ & $0.0183(4)$ & \\
\hline & M3 & $0.44065(18)$ & 0.2500 & $0.78772(17)$ & $0.0193(7)$ & \\
\hline & M4 & $0.44006(4)$ & 0.2500 & $0.78750(4)$ & $0.02066(16)$ & \\
\hline & M5 & $0.43939(5)$ & 0.2500 & $0.78762(7)$ & $0.0197(3)$ & \\
\hline & M6 & $0.43879(15)$ & 0.2500 & $0.78780(16)$ & $0.0182(6)$ & \\
\hline & M7 & $0.43882(10)$ & 0.2500 & $0.78874(11)$ & $0.0192(4)$ & \\
\hline & M8 & $0.43894(5)$ & 0.2500 & $0.79015(6)$ & $0.0216(2)$ & \\
\hline & M9 & $0.43918(6)$ & 0.2500 & $0.79184(8)$ & $0.0189(3)$ & \\
\hline & M10 & $0.43963(8)$ & 0.2500 & $0.79388(9)$ & $0.0184(4)$ & \\
\hline & M11 & $0.44019(8)$ & 0.2500 & $0.79587(10)$ & $0.0173(3)$ & \\
\hline \multirow[t]{11}{*}{$\mathrm{X} 1$} & M1 & $0.6894(3)$ & 0.2500 & $0.6960(3)$ & $0.0236(8)$ & $\mathrm{Br}_{1}$ \\
\hline & M2 & $0.6890(2)$ & 0.2500 & $0.6957(3)$ & $0.0210(10)$ & $\mathrm{Br}_{0.815(18)} \mathrm{Cl}_{0.185(18)}$ \\
\hline & M3 & $0.6882(5)$ & 0.2500 & $0.6958(6)$ & $0.014(2)$ & $\mathrm{Br}_{0.54(4)} \mathrm{Cl}_{0.46(4)}$ \\
\hline & M4 & $0.68820(15)$ & 0.2500 & $0.69496(17)$ & $0.0223(6)$ & $\mathrm{Br}_{0.450(9)} \mathrm{Cl}_{0.550(9)}$ \\
\hline & M5 & $0.6879(2)$ & 0.2500 & $0.6949(4)$ & $0.0232(11)$ & $\mathrm{Br}_{0.289(14)} \mathrm{Cl}_{0.711(14)}$ \\
\hline & M6 & $0.6886(8)$ & 0.2500 & $0.6924(10)$ & $0.024(3)$ & $\mathrm{Br}_{0.15(3)} \mathrm{Cl}_{0.85(3)}$ \\
\hline & M7 & $0.6873(6)$ & 0.2500 & $0.6936(7)$ & $0.027(2)$ & $\mathrm{Br}_{0.14(2)} \mathrm{Cl}_{0.86(2)}$ \\
\hline & M8 & $0.6890(3)$ & 0.2500 & $0.6930(4)$ & $0.0259(13)$ & $\mathrm{Br}_{0.043(12)} \mathrm{Cl}_{0.957(12)}$ \\
\hline & M9 & $0.6892(3)$ & 0.2500 & $0.6931(6)$ & $0.0219(16)$ & $\mathrm{Br}_{0.020(16)} \mathrm{Cl}_{0.980(16)}$ \\
\hline & M10 & $0.6895(5)$ & 0.2500 & $0.6934(7)$ & $0.0214(12)$ & $\mathrm{Cl}_{1}$ \\
\hline & M11 & $0.6897(5)$ & 0.2500 & $0.6940(7)$ & $0.0219(13)$ & $\mathrm{Cl}_{1}$ \\
\hline \multirow[t]{8}{*}{$\mathrm{X} 2$} & M1 & $0.6291(4)$ & 0.2500 & $0.0752(3)$ & $0.0295(10)$ & $\mathrm{Br}_{1}$ \\
\hline & M2 & $0.6301(3)$ & 0.2500 & $0.0750(3)$ & $0.0284(7)$ & $\mathrm{Br}_{1}$ \\
\hline & M3 & $0.6293(6)$ & 0.2500 & $0.0747(5)$ & $0.0318(15)$ & $\mathrm{Br}_{1}$ \\
\hline & M4 & $0.63057(12)$ & 0.2500 & $0.07453(13)$ & $0.0292(5)$ & $\mathrm{Br}_{0.934(9)} \mathrm{Cl}_{0.066(9)}$ \\
\hline & M5 & $0.63077(18)$ & 0.2500 & $0.0735(2)$ & $0.0289(8)$ & $\mathrm{Br}_{0.907(13)} \mathrm{Cl}_{0.093(13)}$ \\
\hline & M6 & $0.6302(5)$ & 0.2500 & $0.0741(6)$ & $0.0259(19)$ & $\mathrm{Br}_{0.81(3)} \mathrm{Cl}_{0.19(3)}$ \\
\hline & M7 & $0.6314(4)$ & 0.2500 & $0.0733(4)$ & $0.0298(14)$ & $\mathrm{Br}_{0.77(2)} \mathrm{Cl}_{0.23(2)}$ \\
\hline & M8 & $0.6320(2)$ & 0.2500 & $0.0745(2)$ & $0.0308(9)$ & $\mathrm{Br}_{0.553(13)} \mathrm{Cl}_{0.447(13)}$ \\
\hline
\end{tabular}

Table 3. Atomic coordinates and displacement parameters for mendipite-related compounds. 
Table 3. Continued.

\begin{tabular}{lllllll}
\hline Atom & Sample & $x$ & $y$ & $z$ & $U_{\text {eq }}, \AA^{2}$ & Occupancy \\
\hline \multirow{4}{*}{ M9 } & $0.6328(3)$ & 0.2500 & $0.0747(4)$ & $0.0287(12)$ & $\mathrm{Br}_{0.369(16)} \mathrm{Cl}_{0.631(16)}$ \\
M10 & $0.6337(5)$ & 0.2500 & $0.0774(6)$ & $0.0312(19)$ & $\mathrm{Br}_{0.186(19)} \mathrm{Cl}_{0.814(19)}$ \\
O11 & $0.6357(6)$ & 0.2500 & $0.0811(7)$ & $0.0295(15)$ & $\mathrm{Cl}_{1}$ \\
O & M1 & $0.5778(15)$ & $-0.006(2)$ & $0.3908(12)$ & $0.021(4)$ & \\
M2 & $0.5800(9)$ & $-0.0053(19)$ & $0.3916(11)$ & $0.013(2)$ & \\
M3 & $0.5830(19)$ & $-0.002(5)$ & $0.3916(19)$ & $0.015(5)$ & \\
M4 & $0.5815(4)$ & $-0.0049(9)$ & $0.3916(5)$ & $0.0184(12)$ & \\
M5 & $0.5828(6)$ & $-0.0079(16)$ & $0.3921(8)$ & $0.0190(19)$ & \\
M6 & $0.5840(15)$ & $-0.005(4)$ & $0.3956(17)$ & $0.014(5)$ & \\
M7 & $0.5830(11)$ & $-0.002(2)$ & $0.3906(12)$ & $0.020(3)$ & \\
M8 & $0.5823(6)$ & $-0.0047(15)$ & $0.3907(7)$ & $0.0218(18)$ & \\
M9 & $0.5814(6)$ & $-0.0039(16)$ & $0.3903(9)$ & $0.021(2)$ & \\
M10 & $0.5810(9)$ & $-0.005(2)$ & $0.3900(10)$ & $0.016(3)$ & \\
M11 & $0.5793(9)$ & $-0.0052(15)$ & $0.3873(11)$ & $0.014(3)$ & \\
\hline
\end{tabular}

factors. Each was refined on the basis of $F^{2}$ for all unique reflections. The final atomic coordinates and anisotropic displacement parameters are given in Table 3, and selected interatomic distances are in Table 4.

\section{Results}

The crystal structure (Fig. 1a) of mendipite-type phases is based upon $\left[\mathrm{O}_{2} \mathrm{~Pb}_{3}\right]^{2+}$ double chains of edge-sharing $\mathrm{OPb}_{4}$ tetrahedra. The $\left[\mathrm{O}_{2} \mathrm{~Pb}_{3}\right]^{2+}$ double chains extend along the $b$ axis (Fig. 1b). Such chains have previously been ob-
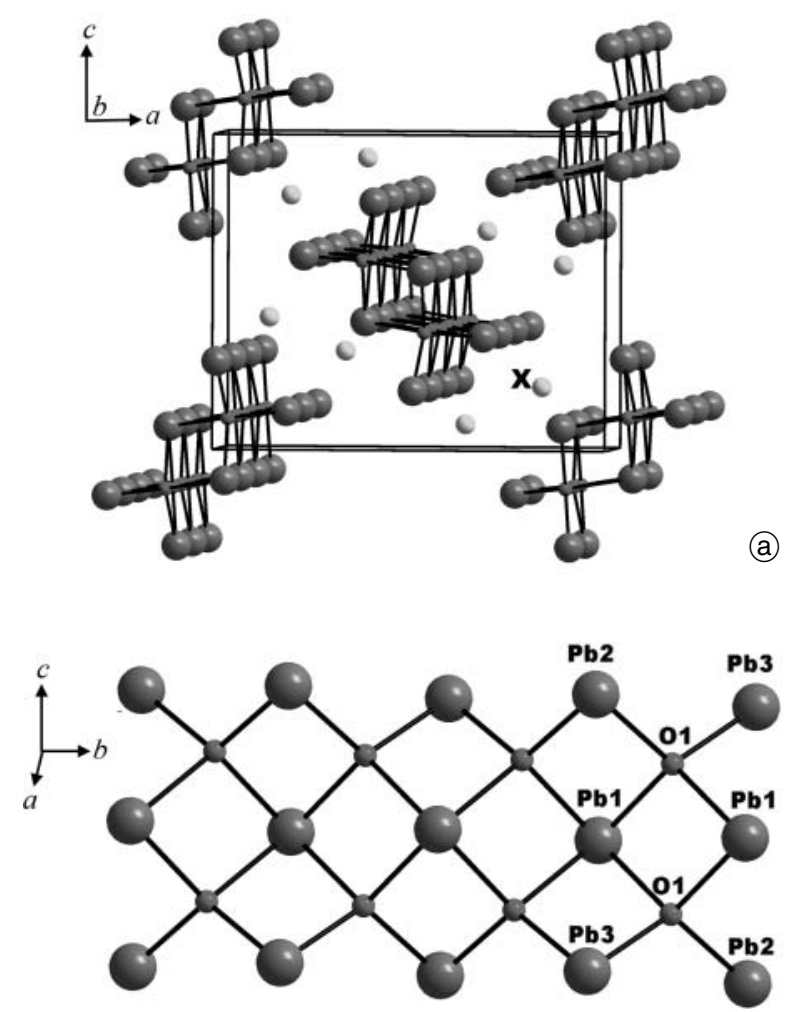

(b)

Fig. 1. Projection of the crystal structure of the mendipite-related compounds. Only the $\mathrm{Pb}-\mathrm{O}$ bonds are shown. $\mathrm{Pb}-$ large dark circles, $\mathrm{X}(\mathrm{X}=\mathrm{Cl}, \mathrm{Br})$ - light circles, $\mathrm{O}$ - small grey circles (a). $\left[\mathrm{O}_{2} \mathrm{~Pb}_{3}\right]^{2+}$ double chain of $\mathrm{OPb}_{4}$ oxocentered tetrahedra shown in balland-stick representation (b). served in the crystal structures of many natural and synthetic lead oxocentered compounds (Krivovichev et al., 2004; Siidra et al., 2007e). In crystal structures of the studied compounds, the chains occur in two mutually perpendicular orientations with the angle varying from $82.48(12)^{\circ}$ (M11) to $89.36(15)^{\circ}$ (M6). The halogen ions connect the chains through weak $\mathrm{Pb}-\mathrm{X}(\mathrm{X}=\mathrm{Cl}, \mathrm{Br})$ bonds only.

There are three symmetrically independent $\mathrm{Pb}^{2+}$ cations in the structures of the mendipite series $\mathrm{Pb}_{3} \mathrm{O}_{2} \mathrm{Cl}_{2}-$ $\mathrm{Pb}_{3} \mathrm{O}_{2} \mathrm{Br}_{2}$. The number of nonequivalent halogen sites is two $(\mathrm{X} 1, \mathrm{X} 2)$. $\mathrm{Pb} 1$ is coordinated by four $\mathrm{O}$ atoms, two $\mathrm{X} 1$ and one $\mathrm{X} 2$ halogen atoms. $\mathrm{Pb} 2$ is coordinated by two
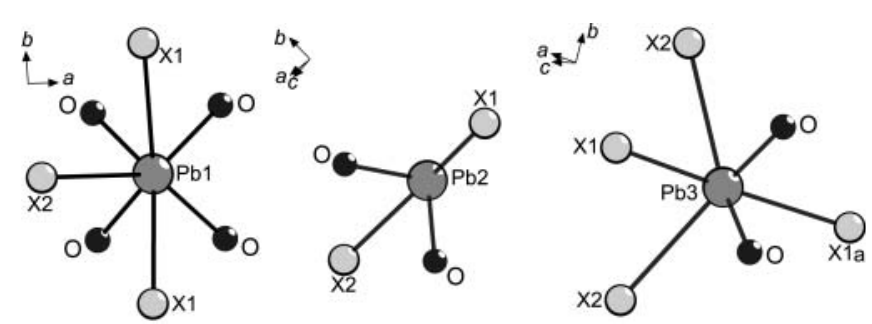

Fig. 2. Coordination of $\mathrm{Pb}$ atoms in the structures of the mendipite series $\mathrm{Pb}_{3} \mathrm{O}_{2} \mathrm{Cl}_{2}-\mathrm{Pb}_{3} \mathrm{O}_{2} \mathrm{Br}_{2}$.

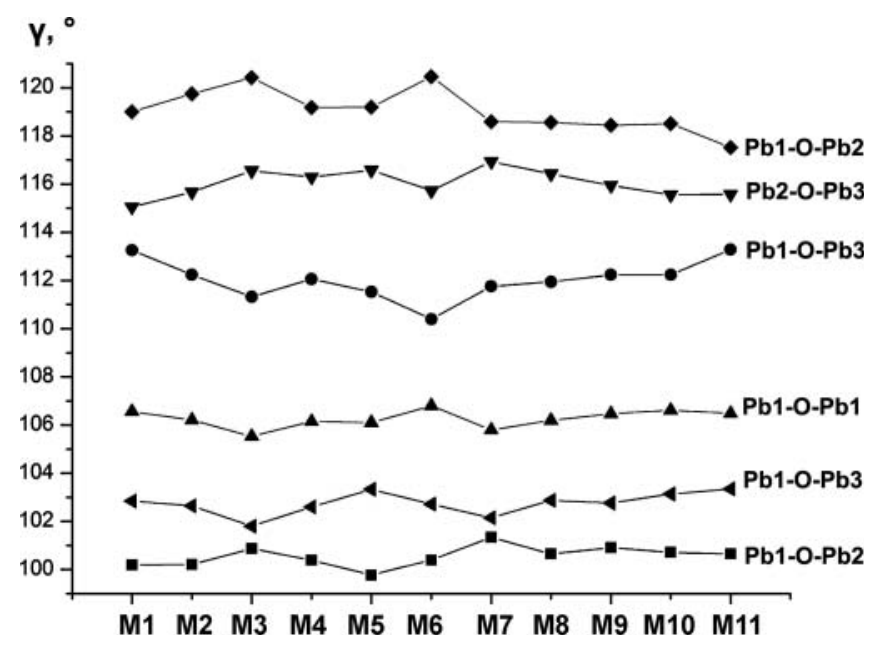

Fig. 3. Variation of angles in the $\mathrm{OPb}_{4}$ tetrahedra in the crystal structures of the mendipite series $\mathrm{Pb}_{3} \mathrm{O}_{2} \mathrm{Cl}_{2}-\mathrm{Pb}_{3} \mathrm{O}_{2} \mathrm{Br}_{2}$. 
Table 4. Selected interatomic distances $(\AA)$ for mendipite-related compounds.

\begin{tabular}{|c|c|c|c|c|c|c|}
\hline Sample & $\mathrm{Pb} 1-\mathrm{O} 12 \mathrm{x}$ & $\mathrm{Pb} 1-\mathrm{O} 12 \mathrm{x}$ & $\mathrm{Pb} 1-\mathrm{X} 12 \times$ & $\mathrm{Pb} 1-\mathrm{X} 2$ & $\mathrm{~Pb} 2-\mathrm{X} 1$ & $\mathrm{~Pb} 2-\mathrm{O} 12 \times$ \\
\hline M1 & $2.335(13)$ & $2.455(17)$ & $3.430(2)$ & $3.574(5)$ & $3.226(4)$ & $2.244(16)$ \\
\hline M2 & $2.335(11)$ & $2.467(11)$ & $3.4224(16)$ & $3.548(3)$ & $3.212(3)$ & $2.224(11)$ \\
\hline M3 & $2.35(2)$ & $2.47(2)$ & $3.402(4)$ & $3.525(7)$ & $3.191(6)$ & $2.18(3)$ \\
\hline M4 & $2.340(5)$ & $2.462(5)$ & $3.3946(9)$ & $3.4953(15)$ & $3.1662(17)$ & $2.219(5)$ \\
\hline M5 & $2.328(9)$ & $2.476(8)$ & $3.3819(16)$ & $3.470(2)$ & $3.146(4)$ & $2.225(8)$ \\
\hline M6 & $2.309(19)$ & $2.46(2)$ & $3.366(5)$ & $3.458(6)$ & $3.107(10)$ & $2.20(2)$ \\
\hline M7 & $2.350(12)$ & $2.449(14)$ & $3.354(4)$ & $3.440(5)$ & $3.113(7)$ & $2.199(14)$ \\
\hline M8 & $2.338(7)$ & $2.451(8)$ & $3.3591(19)$ & $3.422(3)$ & $3.107(4)$ & $2.213(8)$ \\
\hline M9 & $2.338(9)$ & $2.446(8)$ & $3.360(2)$ & $3.412(4)$ & $3.112(5)$ & $2.217(8)$ \\
\hline M10 & $2.327(10)$ & $2.446(11)$ & $3.354(3)$ & $3.392(6)$ & $3.113(6)$ & $2.217(11)$ \\
\hline M11 & $2.326(10)$ & $2.421(10)$ & $3.333(4)$ & $3.340(7)$ & $3.106(7)$ & $2.213(10)$ \\
\hline$\langle\mathrm{Pb}-\mathrm{O}, \mathrm{X}\rangle$ & 2,33 & 2,45 & 3,38 & 3,46 & 3,15 & 2,21 \\
\hline Sample & $\mathrm{Pb} 2-\mathrm{X} 2$ & $\mathrm{~Pb} 3-\mathrm{O} 12 \times$ & $\mathrm{Pb} 3-\mathrm{X} 1$ & Pb3-X1a & $\mathrm{Pb} 3-\mathrm{X} 22 \times$ & \\
\hline M1 & $3.047(4)$ & $2.277(13)$ & $3.097(4)$ & $3.160(4)$ & $3.343(2)$ & \\
\hline M2 & $3.057(3)$ & $2.286(11)$ & $3.088(3)$ & $3.146(3)$ & $3.3455(17)$ & \\
\hline M3 & $3.065(6)$ & $2.29(3)$ & $3.059(7)$ & $3.127(7)$ & $3.330(4)$ & \\
\hline M4 & $3.0836(13)$ & $2.275(5)$ & $3.0402(18)$ & $3.1238(18)$ & $3.3327(7)$ & \\
\hline M5 & $3.103(2)$ & $2.265(9)$ & $3.018(3)$ & $3.111(3)$ & $3.3290(13)$ & \\
\hline M6 & $3.100(5)$ & $2.298(19)$ & $2.989(10)$ & $3.119(10)$ & $3.314(3)$ & \\
\hline M7 & $3.090(4)$ & $2.269(13)$ & $3.002(7)$ & $3.101(7)$ & $3.308(3)$ & \\
\hline M8 & $3.060(2)$ & $2.268(8)$ & $2.978(4)$ & $3.119(4)$ & $3.3010(14)$ & \\
\hline M9 & $3.032(4)$ & $2.276(9)$ & $2.980(4)$ & $3.125(4)$ & $3.2963(19)$ & \\
\hline M10 & $2.976(5)$ & $2.278(10)$ & $2.977(6)$ & $3.123(6)$ & $3.276(3)$ & \\
\hline M11 & $2.892(7)$ & $2.251(10)$ & $2.960(7)$ & $3.100(7)$ & $3.243(4)$ & \\
\hline$\langle\mathrm{Pb}-\mathrm{O}, \mathrm{X}\rangle$ & 3.05 & 2.28 & 3.02 & 3.12 & 3.31 & \\
\hline
\end{tabular}

$\mathrm{O}$ atoms, one $\mathrm{X} 1$ and one $\mathrm{X} 2$ atom. $\mathrm{Pb} 3$ is coordinated by two $\mathrm{O}$ atoms, two $\mathrm{X} 1$ atoms and two $\mathrm{X} 2$ atoms.

Coordination polyhedra for the $\mathrm{Pb}$ atoms are shown in Fig. 2. The short $\mathrm{Pb}-\mathrm{O}$ bonds located on one side of the $\mathrm{Pb}^{2+}$ cations have lengths from $2.18(\mathrm{~Pb} 2-\mathrm{O} 1$ in $\mathrm{M} 3)$ to $2.48 \AA(\mathrm{Pb} 1-\mathrm{O} 1$ in $\mathrm{M} 5)$ with the average $\langle\mathrm{Pb}-\mathrm{O}\rangle$ value of $2.32 \AA$, which is in a good agreement with the values suggested by Krivovichev and Filatov (2001). The average
$\langle\mathrm{Pb}-\mathrm{O}-\mathrm{Pb}\rangle$ angles vary from $109.41^{\circ}$ (M5) to $109.48^{\circ}$ (M1), which is very close to the value of $109.5^{\circ}$ for a regular tetrahedron. The reduction of one or several $\mathrm{Pb}-\mathrm{O}-\mathrm{Pb}$ angle values caused by edge sharing is compensated by increase of the other bond angles (Fig. 3). Average distances between the lead atoms within the $\mathrm{OPb}_{4}$ tetrahedra $(\langle\mathrm{Pb} \cdots \mathrm{Pb}\rangle)$ vary from $3.60 \AA(\langle\mathrm{P} 3 \cdots \mathrm{Pb} 1\rangle$, $(\langle\mathrm{Pb} 2 \cdots \mathrm{Pb} 1\rangle)$ to $3.92 \AA(\langle\mathrm{Pb} 1 \cdots \mathrm{Pb} 3\rangle,(\langle\mathrm{Pb} 1 \cdots \mathrm{Pb} 2\rangle)$

Table 5. The $\mathrm{Pb} \cdots \mathrm{Pb}$ distances in $\mathrm{OPb}_{4}$ tetrahedra in the crystal structures of the mendipite series $\mathrm{Pb}_{3} \mathrm{O}_{2} \mathrm{Cl}_{2}-\mathrm{Pb}_{3} \mathrm{O}_{2} \mathrm{Br}_{2}$.

\begin{tabular}{lllllll|l}
\hline Sample & $\mathrm{Pb} 1-\mathrm{Pb} 1, \AA$ & $\mathrm{Pb} 1-\mathrm{Pb} 3, \AA$ & $\mathrm{Pb} 3-\mathrm{Pb} 1, \AA$ & $\mathrm{Pb} 1-\mathrm{Pb} 2, \AA$ & $\mathrm{Pb} 1-\mathrm{Pb} 2, \AA$ & $\mathrm{Pb} 2-\mathrm{Pb} 3, \AA$ & $\langle\mathrm{Pb} \cdots \mathrm{Pb}\rangle, \AA$ \\
\hline M1 & $3.841(8)$ & $3.954(6)$ & $3.605(15)$ & $3.609(9)$ & $3.946(7)$ & $3.814(7)$ & 3.80 \\
$\mathrm{M} 2$ & $3.841(11)$ & $3.947(11)$ & $3.607(9)$ & $3.603(15)$ & $3.943(9)$ & $3.818(8)$ & 3.79 \\
M3 & $3.836(9)$ & $3.935(8)$ & $3.598(8)$ & $3.595(14)$ & $3.931(13)$ & $3.807(12)$ & 3.78 \\
M4 & $3.839(14)$ & $3.930(8)$ & $3.602(10)$ & $3.599(9)$ & $3.932(11)$ & $3.817(11)$ & 3.79 \\
M5 & $3.840(9)$ & $3.921(9)$ & $3.603(11)$ & $3.598(11)$ & $3.927(9)$ & $3.820(11)$ & 3.79 \\
M6 & $3.834(10)$ & $3.912(7)$ & $3.599(12)$ & $3.591(8)$ & $3.918(15)$ & $3.813(9)$ & 3.78 \\
M7 & $3.828(8)$ & $3.907(10)$ & $3.595(9)$ & $3.597(8)$ & $3.912(12)$ & $3.809(10)$ & 3.78 \\
M8 & $3.831(11)$ & $3.913(9)$ & $3.603(11)$ & $3.593(6)$ & $3.913(14)$ & $3.809(13)$ & 3.78 \\
M9 & $3.833(9)$ & $3.922(12)$ & $3.605(7)$ & $3.598(17)$ & $3.913(7)$ & $3.809(7)$ & 3.78 \\
M10 & $3.828(8)$ & $3.923(8)$ & $3.606(10)$ & $3.595(9)$ & $3.906(8)$ & $3.803(9)$ & 3.78 \\
M11 & $3.803(13)$ & $3.903(8)$ & $3.591(8)$ & $3.569(7)$ & $3.881(8)$ & $3.777(16)$ & 3.75 \\
$\langle\mathrm{~Pb} \cdots \mathrm{Pb}\rangle, \AA$ & 3.83 & 3.92 & 3.60 & 3.60 & 3.92 & 3.81 & \\
\hline
\end{tabular}




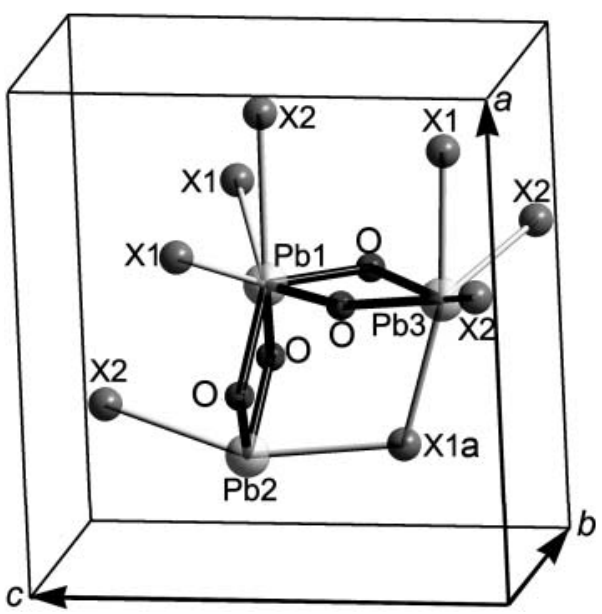

Fig. 4. $\mathrm{Pb}-\mathrm{X}$ and $\mathrm{Pb}-\mathrm{O}$ bonds orientation in the crystal structures of the mendipite series $\mathrm{Pb}_{3} \mathrm{O}_{2} \mathrm{Cl}_{2}-\mathrm{Pb}_{3} \mathrm{O}_{2} \mathrm{Br}_{2}$.

(Table 5). The shortest $\mathrm{Pb} 1 \cdots \mathrm{Pb} 2$ distance between $\left[\mathrm{O}_{2} \mathrm{~Pb}_{3}\right]^{2+}$ chains is $3.59 \AA$ (M11). Edge sharing between two tetrahedra leads to repulsion of oxygen atoms and, as a result, $\mathrm{Pb}-\mathrm{Pb}$ distances become shorter than those corresponding to the unshared edges.

Note the strong shortening of all $\mathrm{Pb} \cdots \mathrm{Pb}$ distances in the structure of the end-member $\mathrm{Pb}_{3} \mathrm{O}_{2} \mathrm{Cl}_{2}$ (Table 5).

The weak $\mathrm{Pb}-\mathrm{X}$ bonds located on the opposite side of the $\mathrm{Pb}^{2+}$ coordination sphere vary from $2.89 \AA(\mathrm{Pb} 2-\mathrm{X} 2$ in $\mathrm{M} 11)$ to $3.57 \AA$ (Pb1-X2 in M1). The evident strong distortion of the $\mathrm{Pb}^{2+}$ coordination polyhedra is due to the stereoactivity of the $6 s^{2}$ lone electron pairs of the $\mathrm{Pb}^{2+}$ cations. The $\mathrm{X} 1$ halogen site is coordinated by five $\mathrm{Pb}^{2+}$ cations, but the $\mathrm{X} 2$ halogen site is coordinated by only four $\mathrm{Pb}^{2+}$ cations. The $\mathrm{Pb}-\mathrm{X}$ bonds are oriented in space as follows (Fig. 4): $\mathrm{Pb} 2-\mathrm{X} 1, \mathrm{~Pb} 2-\mathrm{X} 2$ are oriented along the $c$ axis; $\mathrm{Pb} 1-\mathrm{X} 2, \mathrm{~Pb} 1-\mathrm{X} 1, \mathrm{~Pb} 3-\mathrm{X} 1-$ along the $b$ axis; $\mathrm{Pb} 1-$ $\mathrm{X} 1, \mathrm{~Pb} 3-\mathrm{X} 2$ - along the $b$ axis. The variations for the $\mathrm{Pb}$ $\mathrm{X}$ bonds by the exchange of $\mathrm{Br}$ for $\mathrm{Cl}$ are: $\mathrm{Pb} 1-\mathrm{X} 1$ $\Delta=0.1 ; \mathrm{Pb} 1-\mathrm{X} 2 \quad \Delta=0.23 ; \mathrm{Pb} 2-\mathrm{X} 2 \quad \Delta=0.2 ; \mathrm{Pb} 2-\mathrm{X} 1$ $\Delta=0.12 ; \mathrm{Pb} 3-\mathrm{X} 1 \quad \Delta=0.14 ; \mathrm{Pb} 3-\mathrm{X} 1 \mathrm{a} \Delta=0.06 ; \mathrm{Pb} 3-\mathrm{X} 2$ $\Delta=0.11$ (Table. 4). $\mathrm{Pb} 1-\mathrm{X} 2$ and $\mathrm{Pb} 2-\mathrm{X} 2$ bonds (Fig. 4) are the most sensitive ones to the $\mathrm{X}$ site occupancy, which

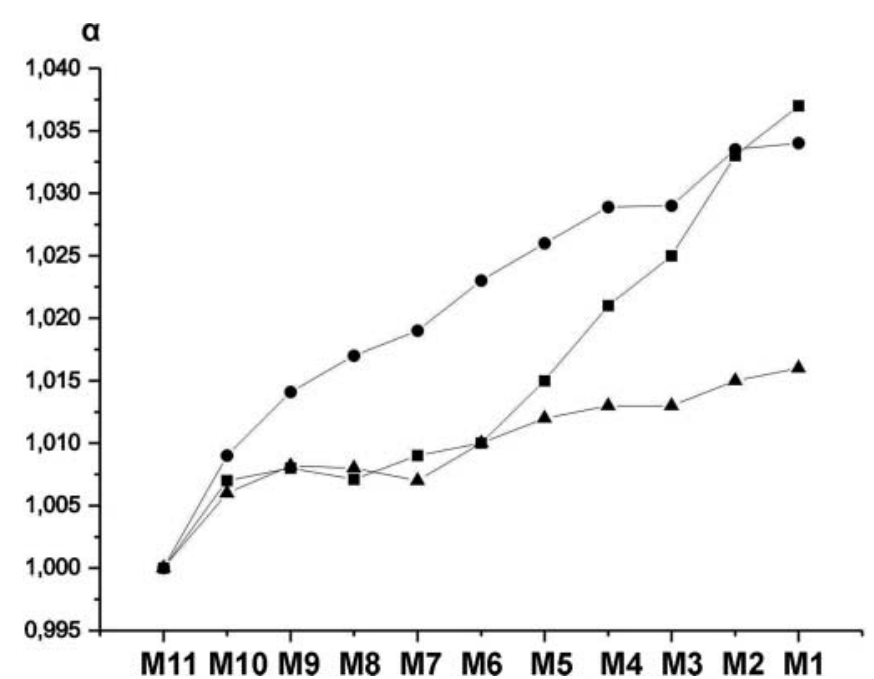

Fig. 5. Normalized lattice parameters $\left(a=a / a_{\mathrm{Cl}}-\boldsymbol{\square}, b / b_{\mathrm{Cl}}-\boldsymbol{\Delta}, c / c_{\mathrm{Cl}}\right.$ - - in the crystal structures of the mendipite series $\mathrm{Pb}_{3} \mathrm{O}_{2} \mathrm{Cl}_{2}-\mathrm{Pb}_{3} \mathrm{O}_{2} \mathrm{Br}_{2}$.

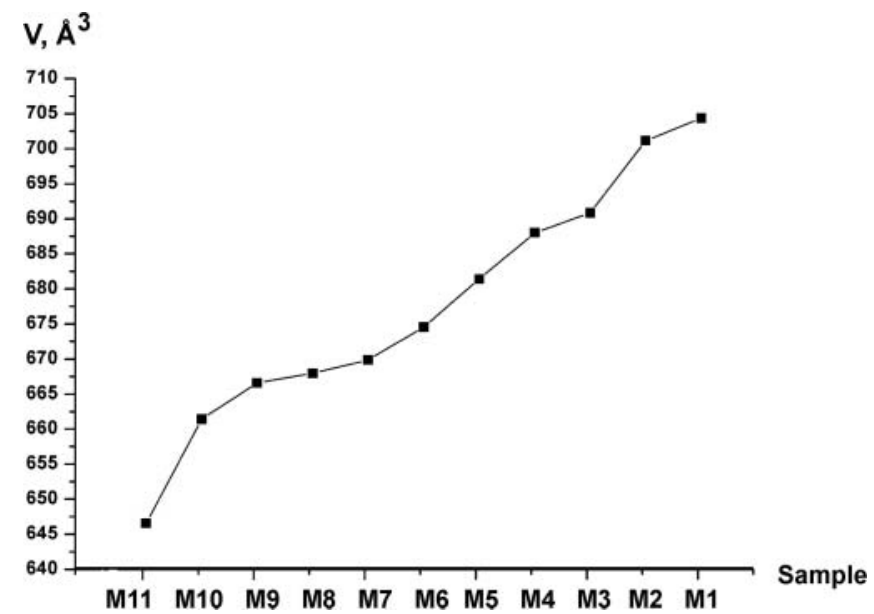

Fig. 6. Cell volume $V, \AA^{3}$ versus the batch number for mendipite-related $\mathrm{Pb}_{3} \mathrm{O}_{2} \mathrm{Cl}_{2}-\mathrm{Pb}_{3} \mathrm{O}_{2} \mathrm{Br}_{2}$ compounds.

is in agreement with the non-linear behavior of the $a$ and $c$ parameters (Fig. 5). The $\mathrm{Pb}-\mathrm{X}$ bonds oriented along the $b$ axis change only slightly.

The unit-cell parameters as determined by single crystal studies showed strong deviation from Vegard's rule (Vegard, Dale, 1928; Urusov, 1992), which can be clearly seen in Fig. 5. Note that the cell volume changes almost linearly (Fig. 6). Nonlinearity of the lattice parameters is caused by selective ordering of the halide anions over two crystallographically nonequivalent sites, X1 and X2 (Table 3 ; Fig. 7). $\mathrm{Br}$ atoms prefer the $\mathrm{X} 2$ position (Table 3), whereas $\mathrm{Cl}$ prefers the $\mathrm{X} 1$ site. $\mathrm{Cl}$ atoms are absent in the $\mathrm{X} 2$ site in M1-M3 samples. In M4-M6 samples, the X2 site is also predominantly occupied by $\mathrm{Br}$ atoms. $\mathrm{Cl}$ begins to prevail in this position only in the three last samples, M9-M11. On the contrary, the $\mathrm{X} 1$ site is preferred by $\mathrm{Cl}$ atoms. It is occupied exclusively by $\mathrm{Cl}$ in $\mathrm{M} 10$, M11 sample.

The $\varphi$ angle between the faces of two adjacent $\mathrm{OPb}_{4}$ tetrahedra (Fig. 8) was determined to analyze the influence of halogen atoms on the structure of the $\left[\mathrm{O}_{2} \mathrm{~Pb}_{3}\right]^{2+}$ chain. From this analysis it is clear that different occupancy of the $\mathrm{X} 1$ site by $\mathrm{Cl}$ or $\mathrm{Br}$ atoms leads to the greatest $\varphi$ angle changes. The $\mathrm{X} 2$ site does not influence the $\left[\mathrm{O}_{2} \mathrm{~Pb}_{3}\right]^{2+}$

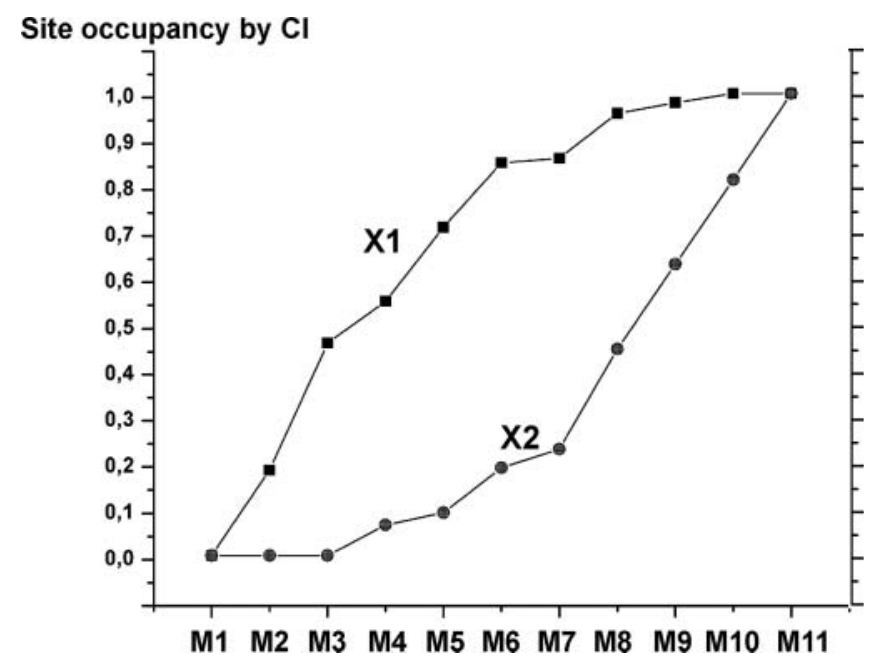

Fig. 7. The $\mathrm{X} 1(\boldsymbol{\square})$ site and $\mathrm{X} 2(\bullet)$ site occupancy by $\mathrm{Cl}$ atoms versus the batch number. 


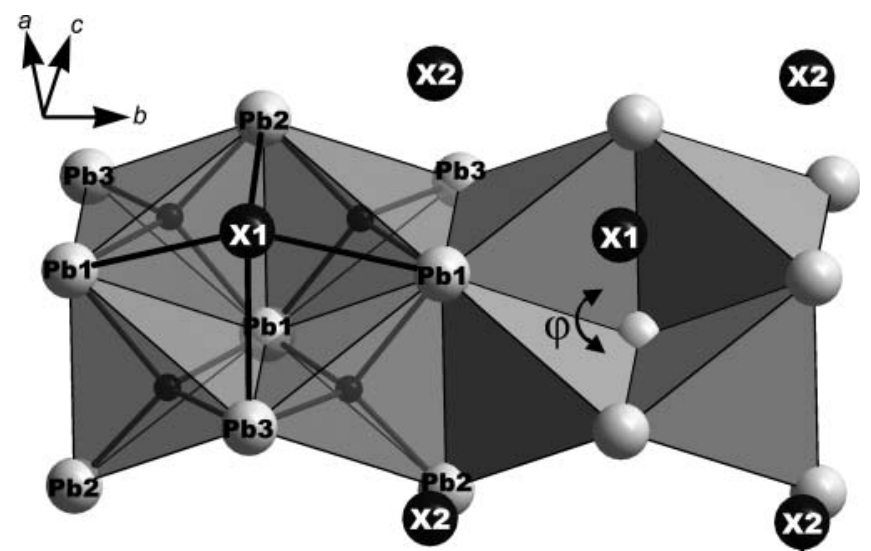

Fig. 8. The $\left[\mathrm{O}_{2} \mathrm{~Pb}_{3}\right]^{2+}$ chain in the crystal structures of the mendipite series $\mathrm{Pb}_{3} \mathrm{O}_{2} \mathrm{Cl}_{2}-\mathrm{Pb}_{3} \mathrm{O}_{2} \mathrm{Br}_{2} . \varphi$ is the angle between the $\mathrm{Pb} 2 \mathrm{~Pb} 1 \mathrm{~Pb} 1$ and $\mathrm{Pb} 3 \mathrm{~Pb} 1 \mathrm{~Pb} 1$ faces of the two adjacent tetrahedra.

chain geometry that much. With the decrease in the $\mathrm{X} 1$ site occupancy (Table 3 ) by the $\mathrm{Br}$ atoms, the $\varphi$ angle increases (Table 6, Fig. 9). Starting from the M7 composition, the $\varphi$ angle begins to decrease, reaching $71.102(5)^{\circ}$ in M11 that contains $\mathrm{Cl}$ atoms only, thus "closing" or "pulling together" the square unit formed by the four adjacent tetrahedra of the $\left[\mathrm{O}_{2} \mathrm{~Pb}_{3}\right]^{2+}$ chain. These observations may be interpreted as a conformation of the $\left[\mathrm{O}_{2} \mathrm{~Pb}_{3}\right]^{2+}$ double chains in the crystal structures of the mendipite series compounds. The $\left[\mathrm{O}_{2} \mathrm{~Pb}_{3}\right]^{2+}$ double chains obviously adapt their geometry to the size of the $\mathrm{X}$ atom. $\mathrm{Br}$ or $\mathrm{Cl}$ ions in the $\mathrm{X} 1$ site are attached to the square unit as shown in Fig. 8 and "stretching" or "pulling together" this unit, accordingly. This mechanism resembles the scheme of adaptation of $\mathrm{XA}_{2}(\mathrm{X}=\mathrm{O}, \mathrm{N} ; \mathrm{A}=$ metal $)$ single anion-centered tetrahedral chains to the large halide ions in the crystal structures of some Ln nitro- and oxyhalides suggested by Krivovichev and Filatov (1998). From the M7 sample, the $\mathrm{X} 1$ site becomes essentially chlorine-rich (Table 3), which leads to the "closing" of the square unit formed by the four adjacent $\mathrm{OPb}_{4}$ tetrahedra. Occupancy of the $\mathrm{X} 1$ site by bromine atoms becomes smaller than 0.15 (Table 3).

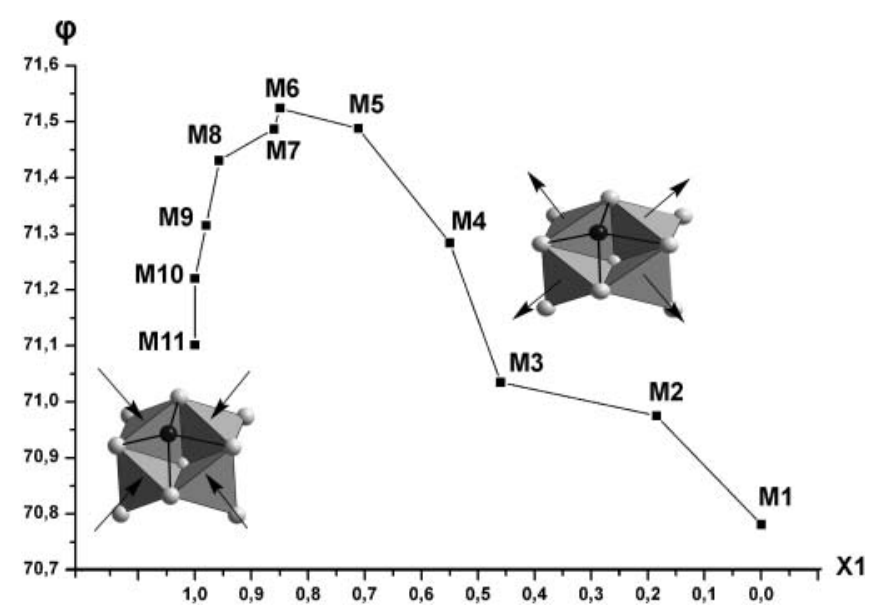

Fig. 9. The $\varphi$ angle versus the $\mathrm{X} 1$ site occupancy in the crystal structures of the mendipite series $\mathrm{Pb}_{3} \mathrm{O}_{2} \mathrm{Cl}_{2}-\mathrm{Pb}_{3} \mathrm{O}_{2} \mathrm{Br}_{2}$. The $\varphi$ angle increases by the reduction of $\mathrm{X} 1$ site occupancy by the $\mathrm{Br}$ atoms, thus "opening" the square unit formed by the four adjacent tetrahedra in a $\left[\mathrm{O}_{2} \mathrm{~Pb}_{3}\right]^{2+}$ chain. Starting from the M7 synthesis the value of $\varphi$ angle again decreases, thus "pulling together" the unit. (See the text for details).
Thus, since the M7 composition, the crystal structure becomes less "strained", which leads to the decrease in the $\varphi$ angle.

Acknowledgments. This work was financially supported by the Alexander von Humboldt Stiftung, RFBR-DFG (07-05-91557), and the Swiss Science Foundation (grant on Crystal Chemistry of Minerals to T.A.). The Russian group thanks the Ministry of Science and Education (Grant RNP 2.1.1.3077) and the Federal Agency on Education (SPbSU innovation project "Innovation educational environment in the classic University") for financial and instrumental support.

\section{References}

Berdonosov, P. S.; Dolgikh, V. A.; Popovkin, B. A.: Structural characterization of lead (II) oxybromide $\mathrm{Pb}_{3} \mathrm{O}_{2} \mathrm{Br}_{2}$. Mater. Res. Bull. 31 (1996) 717-722.

Gabrielson, O.: The crystal structure of mendipite, $\mathrm{Pb}_{3} \mathrm{O}_{2} \mathrm{Cl}_{2}$ Arkiv. Miner. Geol. 2 (1957) 299-304.

Keller, H. L.: Eine neuartige Blei-Sauerstoff-Baugruppe: $\left(\mathrm{Pb}_{8} \mathrm{O}_{4}\right)^{8+}$. Angew. Chem. 95 (1983) 318-319.

Krivovichev, S. V.; Burns, P. C.: Crystal chemistry of lead oxide chlorides. I. Crystal structures of synthetic mendipite, $\mathrm{Pb}_{3} \mathrm{O}_{2} \mathrm{Cl}_{2}$, and synthetic damaraite, $\mathrm{Pb}_{3} \mathrm{O}_{2}(\mathrm{OH}) \mathrm{Cl}$. Eur. J. Mineral. 13 (2001a) 801-809.

Krivovichev, S. V.; Burns, P. C.: Crystal chemistry of lead oxide chlorides. II. Crystal structure of $\mathrm{Pb}_{7} \mathrm{O}_{4}(\mathrm{OH})_{4} \mathrm{Cl}_{2}$. Eur. J. Mineral. 13 (2002) 135-139.

Krivovichev, S. V.; Burns, P. C.: The crystal structure of $\mathrm{Pb}_{8} \mathrm{O}_{5}(\mathrm{OH})_{2} \mathrm{Cl}_{4}$, a synthetic analogue of blixite? Can. Mineral. 44 (2006) 515-522.

Krivovichev, S. V.; Burns, P. C.: Crystal structure of $\mathrm{Pb}_{3} \mathrm{O}_{2}(\mathrm{OH}) \mathrm{Br}$, a Br-analogue of damaraite. Solid State Sci. 3 (2001b) 455-459.

Krivovichev, S. V.; Siidra, O. I.; Nazarchuk, E. V.; Burns, P. C.; Depmeier, W.: Exceptional topological complexity of lead oxide blocks in $\mathrm{Pb}_{31} \mathrm{O}_{22} \mathrm{X}_{18}(\mathrm{X}=\mathrm{Br}, \mathrm{Cl})$. Inorg. Chem. 45 (2006) 38463848.

Krivovichev, S. V.; Filatov, S. K.: Crystal chemistry of minerals and inorganic compounds based on complexes of anion-centered tetrahedra. St. Petersburg University Press, St. Petersburg 2001.

Krivovichev, S. V.; Avdontseva, E. Yu.; Burns, P. C.: Synthesis and crystal structure of $\mathrm{Pb}_{3} \mathrm{O}_{2}\left(\mathrm{Se}_{2} \mathrm{O}_{3}\right)$. Z. Anorg. Allg. Chem. 630 (2004) 558-562.

Krivovichev, S. V.; Filatov, S. K.: Conformation of single chains of anion-centered edge-sharing tetrahedra. Z. Kristallogr. 213 (1998) $316-318$.

Noren, L.; Tan, E. S. Q.; Withers, R. L.; Sterns, M.; Rundlof, H.: A neutron, X-ray and electron diffraction study of the structures of $\mathrm{Pb}_{3} \mathrm{O}_{2} \mathrm{X}_{2}(\mathrm{X}=\mathrm{Cl}, \mathrm{Br})$. Mater. Res. Bull. 37 (2002) 1431-1442.

Pasero, M.; Vacchiano, D.: Crystal structure refinement of mendipite, $\mathrm{Pb}_{3} \mathrm{O}_{2} \mathrm{Cl}_{2}$. Neues Jahrb. Miner. Mh. (2000) 563-569.

Post, J. E.; Buseck, P. R.: Quantitative energy-dispersive analysis of lead halide particles from the Phoenix urban aerosol. Environ. Sci. Technology 19 (1985) 682-685.

Riebe, H.-J.; Keller, H. L.: $\mathrm{Pb}_{13} \mathrm{O}_{10} \mathrm{Br}_{6}$, ein neuer Vertreter der Blei(II)-oxidhalodenide. Z. Anorg. Allg. Chem. 571 (1989) 139147.

Sigman, M. B. Jr.; Korgel, B. A.: Strongly birefringent $\mathrm{Pb}_{3} \mathrm{O}_{2} \mathrm{Cl}_{2}$ nanobelts. J. Am. Chem. Soc. 127 (2005) 10089-10095.

Siidra, O. I.; Krivovichev, S. V.; Armbruster, T.; Depmeier W.: Leadrare-earth oxyhalides: syntheses and characterization of $\mathrm{Pb}_{6} \mathrm{LaO}_{7} \mathrm{X}$ $(\mathrm{X}=\mathrm{Cl}, \mathrm{Br})$. Inorg. Chem. 46 (2007a) 1523-1525.

Siidra, O. I.; Krivovichev, S. V.; Depmeier, W.: Crystal chemistry of natural and synthetic lead oxyhalide. I. Crystal structure of $\mathrm{Pb}_{13} \mathrm{O}_{10} \mathrm{Cl}_{6}$. Proc. Russ. Mineral. Soc. 136(2) (2007b) 79-89.

Siidra, O. I.; Krivovichev, S. V.; Depmeier, W.: Crystal structure of the nonstoichiometric compound $\mathrm{Pb}_{2+x} \mathrm{OCl}_{2+2 x}$ and mechanism of its ionic conductivity. Dokl. Phys. Chem. 414 (2007c) 128-131.

Siidra, O. I.; Krivovichev, S. V.; Depmeier, W.: Crystal chemistry of natural and synthetic lead oxyhalide. II. Crystal structure of $\mathrm{Pb}_{7} \mathrm{O}_{4}(\mathrm{OH})_{4} \mathrm{Br}_{2}$. Proc. Russ. Mineral. Soc. 136(6) (2007d) 85-91.

Siidra, O. I.; Krivovichev, S. V.; Filatov, S. K.: Minerals and synthetic $\mathrm{Pb}$ (II) compounds with oxocentered tetrahedra: review and classification. Z. Kristallogr. 223 (2008) 114-125. 
Smith, W. H.: Lead contamination of the roadside ecosystem. J. Air. Pollut. Control Ass. 26 (1976) 753-766.

Sobanska, S.; Ricq, N.; Laboudigue, A.; Guillermo, R.; Bremard, C.; Laureyns, J.; Merlin, J. C.; Wignacourt, J. P.: Microchemical investigations of dust emitted by a lead smelter. Environ. Sci. Technol. 33 (1999) 1334-1339.

Spencer, L. J.; Mountain, E. D.: New lead-copper minerals from the Mendip Hills, Somerset, England. Miner. Mag. 20 (1923) 67-92.

Urusov, V. S.: A geometric model of deviations from Vegard's rule. J. Solid State Chem. 98 (1992) 223-236.
Vegard, L.; Dale, H.: Untersuchungen über Mischkristalle und Legierungen. Z. Kristallogr. 67 (1928) 148-162.

Vincent, H.; Perrault, G.: Structure cristalline de l'oxychlorure de plomb synthetique $\mathrm{Pb}_{3} \mathrm{O}_{2} \mathrm{Cl}_{2}$. Bull. Soc. Fr. Miner. Cristallogr. 94 (1974) 323-331.

Welch, M. D.; Hawthorne, F. C.; Cooper, M. A.; Kurtis Kyser T.: Trivalent iodine in the crystal structure of schwartzembergite, $\mathrm{Pb}^{2+}{ }_{5} \mathrm{I}^{3+} \mathrm{O}_{6} \mathrm{H}_{2} \mathrm{Cl}_{3}$. Can. Mineral. 39 (2001) 785-795.

Wu, C.-Y.; Biswas, P.: Lead species aerosol formation and growth in multicomponent high-temperature environments. Environ. Eng. Sci. 17 (2000) 41-60.

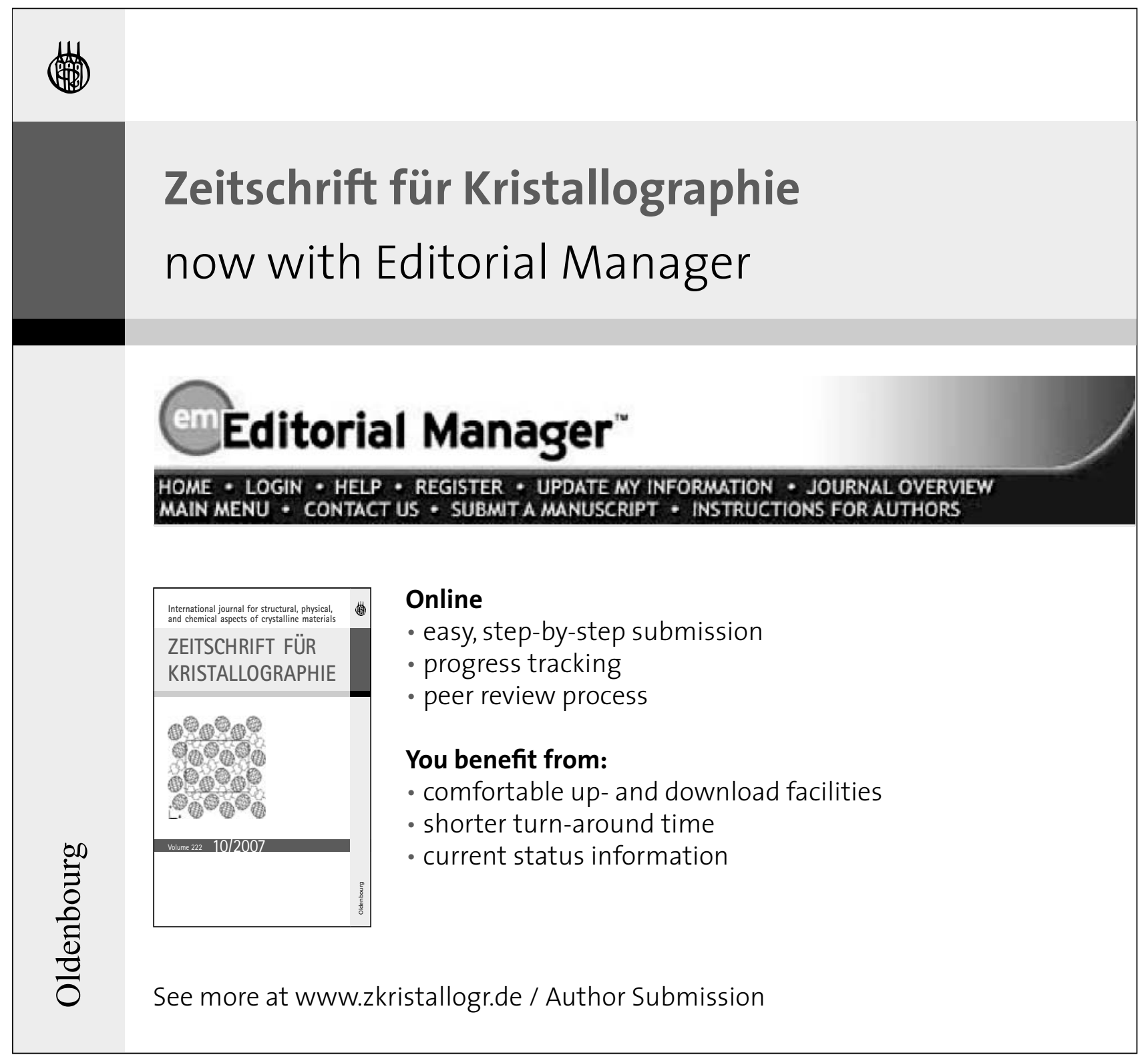

